\title{
Heterogeneous Chip Integration Process for Flexible Wireless Microsystem Application
}

\author{
Tzu-Yuan Chao, Chia-Wei Liang, Y. T. Cheng, Senior Member, IEEE, and Chien-Nan Kuo, Member, IEEE
}

\begin{abstract}
This paper presents a low-cost heterogeneous integration technology combining the previously developed bumpless radio-frequency (RF) system-on-a-package scheme with a special surface cleaning process to assemble a complementary metal-oxide-semiconductor chip with an organic substrate (SU-8/ polydimethylsiloxane) by low-temperature $\mathrm{Au}-\mathrm{Au}$ thermocompressive bonds $\left(<200^{\circ} \mathrm{C}\right)$ for flexible wireless microsystem fabrication. The $\mathrm{RF}$ performance of $-15 \mathrm{~dB}$ return loss and $-0.25 \mathrm{~dB}$ insertion loss at $40 \mathrm{GHz}$ and above $6 \mathrm{MPa}$ bonding strength of a microstrip-to-coplanar-waveguide interconnect transition between the chip and the substrate make the technology practical for flexible wireless microsystem integration.
\end{abstract}

Index Terms-Au-Au thermocompressive (TC) bonding, bumpless interconnecting, flexible electronics, flip-chip, heterogeneous chip integration, surface cleaning.

\section{INTRODUCTION}

$\mathbf{F}$ LEXIBLE electronics has recently become a critical research topic owing to great demand for personal portable devices [1], [2]. To keep the whole process temperature below the glass transition temperature $\left(<200{ }^{\circ} \mathrm{C}\right)$ of flexible substrates, several technologies such as polydimethylsiloxane (PDMS) transfer printing [1], silicon-on-insulator active layer transfer [2], amorphous silicon transistor fabrication [3], organic transistor fabrication [4], and wafer transfer [5] have been developed for the realization of the flexible electronics. The fabrication processes included device substrate thinning, transferring, and backend metal rerouting. The active devices can be fabricated either directly on the flexible substrate or on the device substrate before thinning and transferring onto a flexible substrate. However, poor active device performance, low process flexibility to heterogeneous chip integration, or high manufacturing cost and complexity have resulted in the difficulty in technology implementation in commercial flexible microsystem manufacture. On the other hand, heterogeneous chip assembly schemes have been utilized for multichip module integration on an organic substrate for flexible electronics applications [6]-[9]. Chips fabricated by different process tech-

Manuscript received July 28, 2010; revised November 1, 2010; accepted December 14, 2010. Date of publication January 20, 2011; date of current version February 24, 2011. This work was supported in part by the National Science Council of Taiwan under Grant NSC 99-2220-E-009-065 and in part by the Ministry of Economic Affairs of Taiwan under Grant 99-EC-17-A-03S1-005. The review of this paper was arranged by Editor J. Kanicki.

The authors are with the Microsystems Integration Laboratory, Department of Electronics Engineering and Institute of Electronics, National Chiao Tung University, Hsinchu 300, Taiwan (e-mail: buck34.ee92g@nctu.edu.tw; ytcheng@g2.nctu.edu.tw).

Color versions of one or more of the figures in this paper are available online at http://ieeexplore.ieee.org.

Digital Object Identifier 10.1109/TED.2010.2102357 nologies such as complementary metal-oxide-semiconductor (CMOS), microelectromechanical systems (MEMS), and III-V can be individually optimized and fully integrated onto a flexible substrate to form a microsystem using conventional packaging technology. Although the process temperature of $\mathrm{Cu}$ lateral interconnects [6] and the bonding temperature of flip-chip process using solder bump or anisotropic conductive adhesive [7], [8] can be lower than $200{ }^{\circ} \mathrm{C}$, large structure discontinuity between chips and substrates and high contact resistance will cause a drastic increase in return and insertion loss, respectively, in the interconnecting transition, which is not suitable for high-frequency applications.

Previously, we developed a bumpless interconnecting technology using $\mathrm{Au}-\mathrm{Au}$ thermocompressive (TC) bonds to achieve good RF characteristics up to $50 \mathrm{GHz}$ [10]. Via a selfinterlocking design and flip-chip bonding technique to electrically and mechanically integrate a CMOS chip with a silicon carrier substrate, the interconnect transition can have diminutive parasitic effects to make the transmission of a highfrequency signal with the lowest power loss. Although the technology has shown its great potential in RF system-ona-package integration, high-temperature TC bonding process $\left(>300{ }^{\circ} \mathrm{C}\right.$ ) would make the technology impractical for the chip integration on a flexible substrate. Prior investigation has shown that the cleanness of bonding surface is a key process parameter to lower the bonding temperature [11]. Although Saito et al. has demonstrated a room-temperature surface-activated $\mathrm{Cu}-\mathrm{Cu}$ bonding (SAB) [9] for chip assembly, the SAB has the characteristics of low process uniformity tolerance, long activation time ( $\sim 30 \mathrm{~min}$ ), and special customized tool requirement difficult for the implementation of mass production.

Therefore, in this paper, we will present a bumpless CMOS chip assembly scheme using a low-temperature $\mathrm{Au}-\mathrm{Au} \mathrm{TC}$ bond for flexible wireless microsystem fabrication. A low-cost surface-cleaning process is developed for contaminant removal in bonding interfaces for achieving a low-temperature $\mathrm{Au}-\mathrm{Au}$ bond. Not only CMOS chips but also the other heterogeneous chips such as MEMS and III-V chips can be fully integrated with a flexible organic substrate using the proposed scheme to form a high-performance wireless microsystem.

\section{INTEGRATION PROCESS}

RF CMOS chips are generally designed with microstrips as electrical interconnects for the sake of small form factor. Therefore, a flip-chip transition structure from a coplanar waveguide (CPW) to a microstrip has been extensively utilized for the investigation and design verification of RF packages [12]. The 


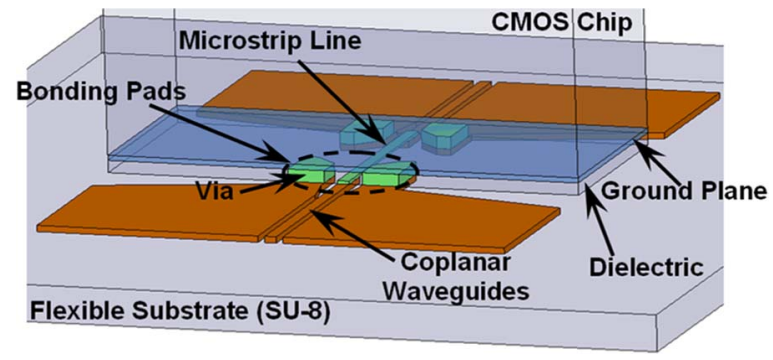

Fig. 1. Scheme of the designed interconnection structure includes two CPWs on flexible substrate and microstrip line on CMOS chip for characterization of the proposed integration technology.

(a)

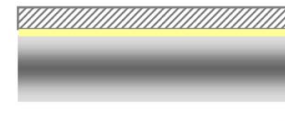

(b)

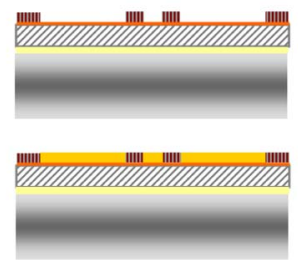

(d)
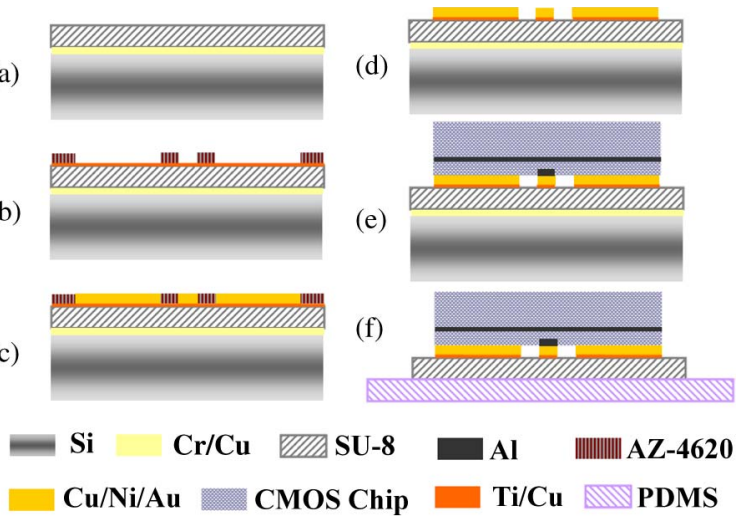

Fig. 2. Scheme of the integration process. (a) Deposition of a $\mathrm{Cr} / \mathrm{Cu}$ sacrificial layer covered with a fully cured SU-8. (b) Ti/Cu seed layer deposition with PR patterning on the top. (c) Cu plating for the fabrication of CPW structure, followed by electroless Ni/Au plating for bonding. (d) PR and seed layer removal. (e) CMOS chip to SU-8 substrate bonding. (f) Si handle wafer detachment by sacrificial layer release in $\mathrm{Cu}$ etchant and then SU-8 film attachment on PDMS.

proposed integration process is demonstrated by a flip-chip transition structure, as shown in Fig. 1, including a microstrip and CPWs, which are designed with a $50-\Omega$ characteristic impedance and $10.5-\mu \mathrm{m}$-wide signal line, and fabricated on a TSMC 0.18- $\mu \mathrm{m}$ RF CMOS chip and an SU-8 flexible substrate, respectively. The integration process, as shown in Fig. 2, begins with the flexible substrate fabrication given here.

Fig. 2(a) shows a silicon substrate that is first sputtered with $10 / 300$-nm-thick $\mathrm{Cr} / \mathrm{Cu}$ as a sacrificial layer, followed by SU-8 spin-coating. The thickness of the SU-8 layer is about $26 \mu \mathrm{m}$. After photopatterning the SU-8, the substrate is hardbaked at $200{ }^{\circ} \mathrm{C}$ for $2 \mathrm{~h}$ in order to make a fully cross-linked SU-8 for having a higher glass transition temperature $\mathrm{Tg}$ [13]. The baking process can enhance TC bonding reliability by preventing the sudden changes in terms of the coefficient of thermal expansion increase and the stiffness decrease in the SU-8 once the bonding temperature is larger than the $T g$ of SU-8. In addition, it can also increase the SU-8's chemical stability in the surface-cleaning process for bonding. After substrate baking, a 10/90-nm Ti/Cu seeding layer is deposited on the SU-8, followed by a photolithograph process using a $10-\mu$ m-thick AZ 4620 photoresist to define the region for the CPW fabrication, as shown in Fig. 2(b). After electroplating an $8-\mu \mathrm{m}$-thick copper CPW on that region, a serial process of $1-\mu \mathrm{m}$ electroless Ni and $0.4-\mu \mathrm{m}$ electroless Au is performed to metallize the $\mathrm{Cu}$ surface for the bonding, as shown in Fig. 2(c). Fig. 2(d) shows that the AZ 4620 and $\mathrm{Ti} / \mathrm{Cu}$ seed layers are
TABLE I

Surface ElEment Content ANALysis By XPS Before AND AFTER ACIDIC SURFACE Cleaning

\begin{tabular}{c|ccc}
\hline \hline \multirow{2}{*}{$\begin{array}{c}\text { Cleaning time } \\
\text { (seconds) }\end{array}$} & \multicolumn{3}{|c}{ Surface Contents (at.\%) } \\
\cline { 2 - 4 } & $\mathrm{C}$ & $\mathrm{O}$ & $\mathrm{Au}$ \\
\hline 0 & 1.71 & 56.38 & 41.91 \\
\hline 180 & 1.45 & 33.95 & 64.4 \\
\hline \hline
\end{tabular}

TABLE II

SPecific Contact Resistance Versus Bonding Temperature

\begin{tabular}{l|ccc}
\hline \hline Temperature $\left({ }^{\circ} \mathrm{C}\right)$ & 160 & 200 & 240 \\
\hline SCR $\left(10^{-7} \Omega \cdot \mathrm{cm}^{2}\right)$ & $5.65 \pm 1.86$ & $4.74 \pm 1.69$ & $2.84 \pm 1.03$ \\
\hline \hline
\end{tabular}

then sequentially removed using ACE, CR-7 T, and then BOE. On the other hand, the contact surfaces of TSMC $0.18-\mu \mathrm{m}$ CMOS chip will be metallized using a maskless doublezincating process, followed by $0.2-\mu \mathrm{m}$ electroless $\mathrm{Ni}$ deposition and $0.4-\mu \mathrm{m}$ electroless Au. Detail CMOS chip preparation for the bumpless integration has been detailed in [10].

Piranha clean has been used as a standard cleaning procedure in CMOS front-end processes [14]. In fact, the piranha clean process can also be utilized in the proposed integration technique for bonding surface cleaning since the Au pads and passivation layer on the CMOS chips in the proposed integration scheme are all chemical inert to the cleaning chemical. Meanwhile, SU-8 is a photopatternable polymer with several superior material properties including good chemical stability, high flexibility, good electromagnetic properties, and waferlevel process capability that make it fascinating as a flexible substrate material. Thus, in the integration scheme, the acidic mixture solution of $\mathrm{H}_{2} \mathrm{SO}_{4}$ and $\mathrm{H}_{2} \mathrm{O}_{2}$ with the volume ratio of $3: 1$ is then employed as a cleanser for realizing low-temperature $\mathrm{Au}-\mathrm{Au}$ TC bonds. Once both surfaces of the CMOS chip and SU-8 substrate are cleaned at about $50{ }^{\circ} \mathrm{C}$, the CMOS chip is flip-chip bonded to the SU-8 substrate at the conditions of $180{ }^{\circ} \mathrm{C}$ and $100-\mathrm{MPa}$ applied pressure for $3 \mathrm{~min}$, as shown in Fig. 2(e). At final, the sacrificial $\mathrm{Cr} / \mathrm{Cu}$ layer is chemically etched away in $\mathrm{Cu}$ etchant (100:5:5 $\mathrm{H}_{2} \mathrm{O}: \mathrm{CH}_{3} \mathrm{COOH}: \mathrm{H}_{2} \mathrm{O}_{2}$ ) to release the SU-8 substrate, which can be directly attached to a PDMS (Sylgard 184) supporting substrate to form a flexible microsystem, as shown in Fig. 2(f).

\section{Results AND Discussion}

The surface-cleaning process for low-temperature $\mathrm{Au}-\mathrm{Au}$ TC bonding is characterized using a daisy chain structure with ten bumps formed within two Si chips. Table I lists X-ray photoelectron spectrometer measurements regarding the surface contents of Au bonding pads before and after the surface-cleaning treatment using the aforementioned cleaning process. The increase in Au content from $41.9 \%$ to $64.6 \%$ and the reduction in carbon and oxygen contents indicate that organic contamination has been effectively removed from the pad surface. Table II lists the measured specific contact resistance (SCR) versus the bonding temperature of $\mathrm{Au}-\mathrm{Au}$ bonds under the conditions of 100-MPa applied pressure for $3 \mathrm{~min}$. For a 4-min cleaning treatment, the bonding temperature can be lowered down to $160{ }^{\circ} \mathrm{C}$ with a $(5.65 \pm 1.86) \times 10^{-7} \Omega \bullet \mathrm{cm}^{2} \mathrm{SCR}$. 
(a)

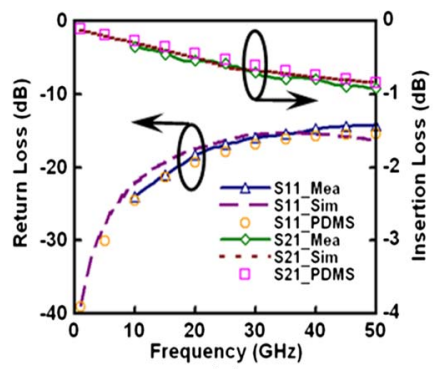

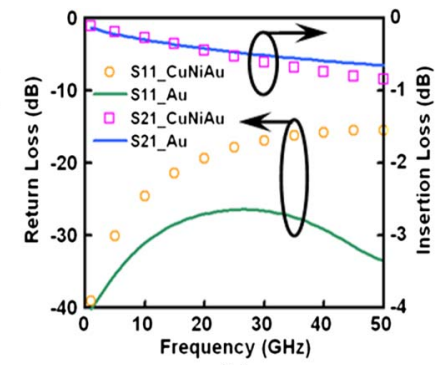

(b)
Fig. 3. (a) Comparison of the RF measurements and HFSS simulations on SU-8/Si and on SU-8/PDMS, respectively, of the designed transition structure. (b) Comparison of the simulation results of the transition on SU-8/PDMS with $\mathrm{CPW}$ made by either $\mathrm{Cu} / \mathrm{Ni} / \mathrm{Au}$ or pure $\mathrm{Au}$, respectively.

Two-port $S$-parameters of the transition structure are measured using Agilent E8364B PNA and Cascade Infinity GSG probe in the frequency range of $10-50 \mathrm{GHz}$. In order to maintain measurement and calibration accuracy, the designed interconnect measurement is performed before handling wafer detachment rather than directly probing on the released SU-8. The measured $S$-parameters have excluded parasitic effects from measurement pads and moved reference planes near the edge of bonding pads using thru-reflect-line calibration. Fig. 3 shows the RF performance of the CPW-microstrip-CPW flipchip transition, i.e., including two interconnect transitions and a microstrip. Better than $-15 \mathrm{~dB}$ return loss and lower than $-0.8 \mathrm{~dB}$ insertion loss up to $40 \mathrm{GHz}$ have been achieved and closely matched with HFSS simulation results, as shown in Fig. 3(a). The measured return loss is as good as that of the microstrip-to-CPW flip-chip compensated interconnects with pure $\mathrm{Au}$ line structures on an $\mathrm{Al}_{2} \mathrm{O}_{3}$ substrate demonstrated by $\mathrm{Wu}$ et al. [12]. In fact, the return loss can be further improved with a better impedance match between the CPW and the microstrip by process optimization in terms of the CPW dimensions and metal thickness control. In addition, according to the simulation results, there is no obvious RF performance difference between the transition on the SU-8 substrate with a Si handle wafer and that with a PDMS substrate, as shown in Fig. 3(a). It can be attributed to the SU-8 layer, which is thick enough to prevent the possible influence of the sacrificial $\mathrm{Cu}$ layers on the performance.

After deducting the loss of microstrip $(-0.31 \mathrm{~dB}$ at $40 \mathrm{GHz})$ from the measured insertion loss $(-0.8 \mathrm{~dB}$ at $40 \mathrm{GHz})$, the loss of a bumpless interconnect transition can be calculated about $-0.25 \mathrm{~dB}$ at $40 \mathrm{GHz}$. Owing to process limitation, electroless $\mathrm{Ni}$ simultaneously acting as the diffusion barrier layer between $\mathrm{Au}$ and $\mathrm{Cu}$ and the seeding layer of electroless $\mathrm{Au}$ is used in the fabrication of the interconnect transition and CPW in our case. Since the electroless Ni has a larger resistivity $(\sim 100 \mu \Omega \bullet \mathrm{cm})$, which will result in a larger ohmic loss in comparison with that of $\mathrm{Cu}(1.7 \mu \Omega \bullet \mathrm{cm})$ and $\mathrm{Au}(2.2 \mu \Omega \bullet \mathrm{cm})$, the insertion loss performance is, therefore, worse than Wu's result $(-0.065 \mathrm{~dB}$ at $40 \mathrm{GHz})$. Fig. 3(b) shows the comparison results of the transition on the SU-8/PDMS substrate with the CPW made by either $\mathrm{Cu}(8 \mu \mathrm{m}) / \mathrm{Ni}(1 \mu \mathrm{m}) / \mathrm{Au}(0.4 \mu \mathrm{m})$ or pure $\mathrm{Au}(9.4 \mu \mathrm{m})$, respectively. The insertion loss of one transition can be reduced down to $-0.14 \mathrm{~dB}$ due to lower metal loss

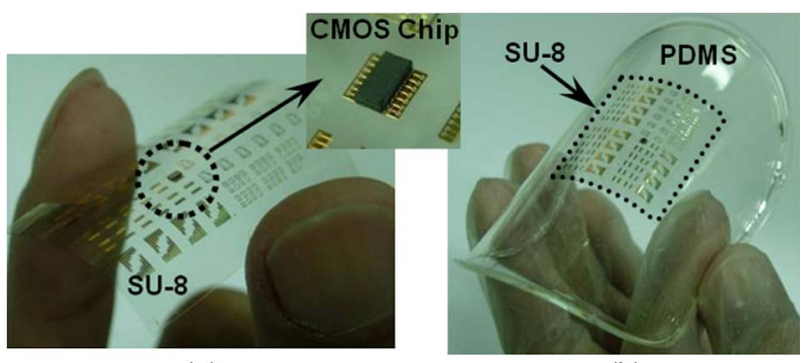

(a)

(b)

Fig. 4. Optical photographs of (a) detached SU-8 film. The enlarged inset photograph shows CMOS chip successfully bonded on SU-8, and (b) SU-8 attaches to and bends along with thick PDMS substrate.

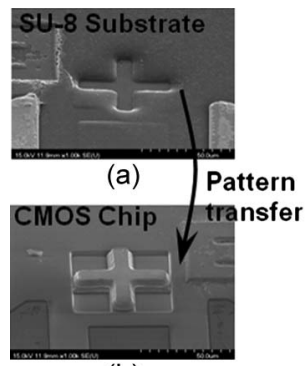

(b)

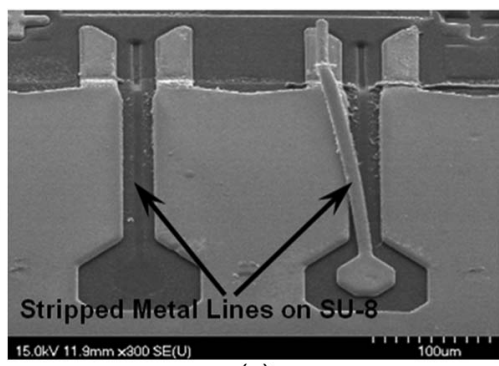

(c)
Fig. 5. Enlarged scanning electron micrographs of two bonded substrates, i.e., CMOS and SU-8, respectively, after forcefully separating the compressive bond. (a) Alignment mark torn away from the SU-8 substrate. (b) Alignment mark transferred onto the CMOS chip. (c) Fully stripped metal lines on the SU-8 substrate.

once the material of $\mathrm{CPW}$ is changed from $\mathrm{Cu} / \mathrm{Ni} / \mathrm{Au}$ to pure $\mathrm{Au}$. Although there is still $-0.075 \mathrm{~dB}$ loss difference between our technology and Wu's work, the first demonstration of the integration of CMOS chip to a flexible substrate with the characteristics of small bonding pad (minimum $10.5 \mu \mathrm{m}$ in width) and simple structure design has revealed the potential of this technology for the integration of high-performance flexible wireless microsystems.

Fig. 4 shows optical photographs of a CMOS chip bonded with the SU-8 film released from a handling silicon wafer and the bonded sample attached to a PDMS film, respectively. The bonding strength is qualitatively characterized by forcefully separating the bonded substrates, as shown in Fig. 5. Either the Au pad is stripped away from the SU- 8 and bonded to the CMOS chip or the Au pad is delaminated directly from the SU-8 after breaking the bonded interface, indicating that the bonding strength can be larger than $6 \mathrm{MPa}$ [15]. Nevertheless, the detail process optimization and characterization of the surface cleaning and flip-chip bonding, and the investigation of the process correlation to the bonding strength and contact resistance are still underway.

\section{CONCLUSION}

In summary, a low-cost heterogeneous chip integration technology using low-temperature $\mathrm{Au}-\mathrm{Au}$ TC bonding has been successfully demonstrated with a good broadband electrical interconnect performance between a TSMC $0.18-\mu \mathrm{m}$ RF CMOS chip and SU-8 polymer substrate. Not only CMOS chips but also the other heterogeneous chips such as MEMS and III-V chips can be fully integrated with a flexible organic substrate 
using the proposed scheme to form a high-performance wireless microsystem.

\section{ACKNOWLEDGMENT}

The authors would like to thank the Chip Implementation Center for the support on complementary metal-oxidesemiconductor chip fabrication and the National Center for High-Performance Computing for the support on the electromagnetic simulator.

\section{REFERENCES}

[1] D.-H. Kim, J.-H. Ahn, H.-S. Kim, K. J. Lee, T.-H. Kim, C.-J. Yu, R. G. Nuzzo, and J. A. Rogers, "Complementary logic gates and ring oscillators on plastic substrates by use of printed ribbons of singlecrystalline silicon," IEEE Electron Device Lett., vol. 29, no. 1, pp. 73-76, Jan. 2008.

[2] S. Srisomrun, Y. Mita, K. Hoshino, M. Sugiyama, and T. Shibata, "Silicon on PDMS: SOI extra thin active layer transferred to organic film for flexible applications," in Proc. IEEE MEMS Conf., Kobe, Japan, 2007, pp. 263-266.

[3] L. Han, K. Song, P. Mandlik, and S. Wagner, "Ultraflexible amorphous silicon transistors made with a resilient insulator," Appl. Phys. Lett., vol. 96, no. 4, p. 042 111, Jan. 2010.

[4] H. Klauk, M. Halik, U. Zschieschang, F. Eder, G. Schmid, and C. Dehm, "Pentacene organic transistors and ring oscillators on glass and on flexible polymeric substrates," Appl. Phys. Lett., vol. 82, no. 23, pp. 4175-4177, Jun. 2003.

[5] L. H. Guo, Q. X. Zhang, H. Y. Li, E. B. Liao, L. K. Bera, W. Y. Loh, C. C. Cheng, G. Q. Lo, N. Balasubramanian, and D. L. Kwong, "Successful transferring of active transistors, RF-passive components and high density interconnect from bulk silicon to organic substrates," in IEDM Tech. Dig., 2005, pp. 675-678.

[6] M. Murugesan, J.-C. Bea, T. Fukushima, T. Konno, K. Kiyoyama, W.-C. Jeong, H. Kino, A. Noriki, K.-W. Lee, T. Tanaka, and M. Koyanagi, "Cu lateral interconnects formed between 100- $\mu$ m-thick self-assembled chips on flexible substrates," in Proc. 59th IEEE ECTC, 2009, pp. 1496-1501.

[7] T. Zhang, Z. Hou, R. W. Johnson, L. Del Castillo, A. Moussessian, R. Greenwell, and B. J. Blalock, "Flexible electronics: Thin silicon die on flexible substrates," IEEE Trans. Electron. Packag. Manuf., vol. 32, no. 4, pp. 291-300, Oct. 2009.

[8] Y.-S. Lin, T.-F. Yang, W.-C. Chen, T.-H. Chen, C.-C. Cheng, and Y.-H. Yeh, "Fine pitch compliant bump interconnection for flip chip on flexible display packaging by anisotropic conductive film," in Proc. 57th IEEE ECTC, 2007, pp. 501-506.

[9] K. Saito, T. Fujii, Y. Akiyama, T. Usami, K. Otsuka, and T. Suga, "Study on the electrical performance of $80 \mu \mathrm{m}$ pitch bumpless bonding for several GHz interconnection," in Proc. 55th IEEE ECTC, 2005, pp. 1127-1131.

[10] T.-Y. Chao, C.-H. Li, Y. C. Chen, H.-Y. Chen, Y.-T. Cheng, and C.-N. Kuo, "An interconnecting technology for RF MEMS heterogeneous chip integration," IEEE Trans. Electron Devices, vol. 57, no. 4, pp. 928938, Apr. 2010.

[11] J. Jellison, "Effect of surface contamination on the thermocompression bondability of gold," IEEE Trans. Parts, Hybrids, Packag., vol. PHP-11, no. 3, pp. 206-211, Sep. 1975.

[12] W.-C. Wu, L.-H. Hsu, E. Y. Chang, C. Karnfelt, H. Zirath, J. P. Starski, and Y.-C. Wu, "60 GHz broadband MS-to-CPW hot-via flip chip interconnects," IEEE Microw. Wireless Compon. Lett., vol. 17, no. 11, pp. 784786, Nov. 2007.

[13] R. Feng and R. J. Farris, "Influence of processing conditions on the thermal and mechanical properties of SU-8 negative photoresist coatings," J. Micromech. Microeng., vol. 13, no. 1, pp. 80-88, Jan. 2003.

[14] J. D. Plummer, M. D. Deal, and P. B. Griffin, Silicon VLSI Technology: Fundamentals, Practice, and Modeling. Englewood Cliffs, NJ: PrenticeHall, 2000.

[15] M. Nordström, A. Johansson, E. S. Noguerón, B. Clausen, M. Calleja, and A. Boisen, "Investigation of the bond strength between the photo-sensitive polymer SU-8 and gold," Microelectron. Eng., vol. 78/79, pp. 152-157, Mar. 2005.

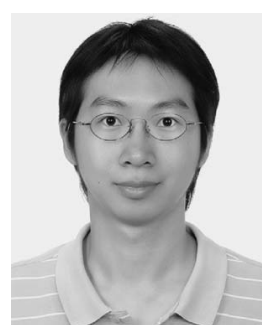

Tzu-Yuan Chao was born in Taiwan. He received the B.S. degree in physics from National Central University, Taoyuan, Taiwan, in 2002. He is currently working toward the Ph.D. degree with the Microsystems Integration Laboratory, Department of Electronics Engineering, National Chiao-Tung University, Hsinchu, Taiwan.

His research interests include nanocomposite material for radio-frequency (RF) integrated circuit applications and the design and fabrication of RF system-on-packaging.

Chia-Wei Liang, photograph and biography not available at the time of publication.

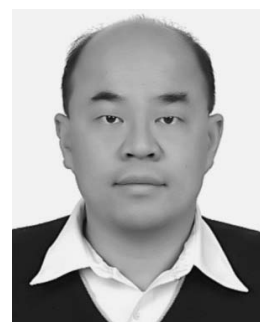

applications

He was in Army service in Taiwan for two years. He was a Research Staff Member with IBM Thomas J. Watson Research Center, Yorktown Heights, where he was involved in several system-on-a-package $(\mathrm{SoP})$ projects. In 2002, he became an Assistant Professor with the Microsystems Integration Laboratory, Department of Electronics Engineering, National Chiao Tung University, Hsinchu, Taiwan, where he has been a Professor since 2009. His research interests include the fundamental study of materials for microsystem integration and nano/MEMS applications, SoP, and the design and fabrication of microsensors and microactuators.

Dr. Cheng is a member of Institute of Physics (IOP) and Phi Tau Phi. He was a corecipient of the 2006 Best Paper Award presented at the 13th IEEE International Conference on Electronics, Circuits and Systems.

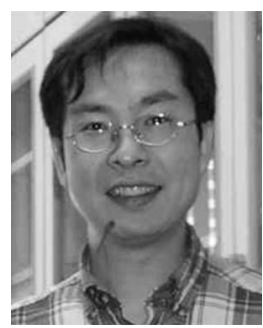

Chien-Nan Kuo (S'93-M'97) received the B.S. degree in electronic engineering from National Chiao Tung University, Hsinchu, Taiwan, in 1988, the M.S. degree in electrical engineering from National Taiwan University, Taipei, Taiwan, in 1990, and the $\mathrm{Ph} . \mathrm{D}$. degree in electrical engineering from the University of California at Los Angeles, Los Angeles, in 1997.

In 1997, he was with ADC Telecommunications, San Diego, CA, as a Member of Technical Staff with the Mobile System Division, where he was involved in wireless base-station design. In 1999, he was with Broadband Innovations Inc., San Diego, CA. In 2001, he was with the Microelectronics Division, IBM. He is currently an Associate Professor with the Microsystems Integration Laboratory, Department of Electronics Engineering, National Chiao Tung University. His research interests include reconfigurable RF circuit and system integration design, low-power design for the application of wireless sensor networks, and development of circuit package co-design in the systemin-package technique.

Dr. Kuo was a recipient of the IEEE MTT Graduate Fellowship Award in 1996. He was a corecipient of the 2006 Best Paper Award presented at the 13th IEEE International Conference on Electronics, Circuits and Systems. He has served as a Program Committee member in the IEEE Asian Solid-State Circuits Conference since 2005 and IEEE Silicon Monolithic Integrated Circuits in RF Systems since 2007. 